\title{
Cooperação Internacional em Ciência, Tecnologia e Inovação na América latina: o Papel do Programa Ibero-Americano de Ciencia y Tecnología PARA EL Desarrollo (Programa CYTED)
}

\author{
Dirceu Yoshikazu Teruya ${ }^{(*)}$ \\ André Carneiro da Cunha Moutinho de Carvalho(*)
}

\begin{abstract}
Resumo: O objetivo deste artigo é verificar a importância relativa do Programa Ibero-americano Ciencia y Tecnología para el Desarrollo (Programa CYTED) como instrumento de articulação dos agentes latino-americanos e de cooperação internacional em matéria de ciência, tecnologia e inovação para países da América Latina. A metodologia será análise das ações do Programa CYTED para o desenvolvimento científico, tecnológico e inovativo dos agentes latinoamericanos. O resultado é que este programa tem uma importância relativa maior em relação aos países menos estruturados e, é necessário um melhor arranjo institucional de cada país como forma de promover uma integração dos instrumentos entre Programa CYTED e as políticas nacionais de cada país.
\end{abstract}

Palavras-chave: Cooperação Internacional, Programa CYTED, Ciência, Tecnologia e Inovação.

Abstract: The objective of this article is to verify the relative importance of Ibero-American Science and Technology for Development (CYTED Program) as a mechanism of articulation among innovative agents and international co-operation in terms of science, technology and innovation in Latin America. The methodology is based on the analysis of the instrument of CYTED Program to promote the technological and innovative development of Ibero-American innovative agents (universities govern and firms). The result is CYTED Program has the relative importance for less structured countries in Latin America in terms of Science, Technology and Innovation. Besides, it is necessary the improvement of the institutional arrangement of each country of Latin America to promote the integration between the instrument of CYTED Program and national policies.

Keywords: International Co-operation, CYTED Program, Science, Technology and Innovation.

(*) Pesquisador em Propriedade Industrial do Instituto Nacional da Propriedade Industrial (INPI), Brasil. E-mail: teruya@inpi.gov.br.

(**) Mestrando em propriedade intelectual e Inovação e Analista da Financiadora de Estudos e Projetos (FINEP). E-mail: acarval@finep.gov.br. Recebido em:27.2.2010 e aceito em: 26.4.2010. 


\section{INTRODUÇÃO}

O processo de desenvolvimento científico, tecnológico e inovativo tem se tornado cada vez mais complexo, exigindo uma articulação eficiente dos agentes envolvidos, a saber, universidades, governo e firmas. O ciclo tecnológico e de produtos está cada vez menor, o investimento em Pesquisa e Desenvolvimento — P\&D tem sido cada vez maior, com aumento do risco e a necessidade de ser competitivo no mercado internacional. Para tanto, os agentes envolvidos necessitam de um background mínimo para o desenvolvimento de competências tecnológicas, bem como linhas de financiamento de longo prazo para que possam investir nas atividades de Pesquisa e Desenvolvimento - P\&D.

Dessa forma, as interações entre estes agentes são fundamentais para a difusão do conhecimento e a promoção da inovação tecnológica nas firmas. Em países em desenvolvimento, como é o caso dos países da América Latina, e em países cujas relações entre estes agentes são consideradas imaturas, a cooperação internacional científica, tecnológica e inovativa pode ser um meio para intensificar as relações entre os agentes, no sentido de minimizar os riscos inerentes ao processo de inovação, bem como otimizar os escassos recursos financeiros e técnicos para o desenvolvimento e/ou aperfeiçoamento de produtos e/ou processos industriais.

Os agentes do processo científico, tecnológico e inovativo na América Latina, de um modo geral, apresentam uma capacidade limitada de criação de tecnologia, decorrente de um background insuficiente para o desenvolvimento tecnológico endógeno por si só. Além disso, esses países apresentam restrições institucionais e macroeconômicas, que limitam o seu processo de desenvolvimento tecnológico, e sua atuação efetiva como um player significativo na produção de conhecimento tecnológico e inovativo.

Assim, a cooperação internacional em ciência, tecnologia e inovação entre os agentes dos países latino-americanos constitui um instrumento importante para o aprendizado, difusão da tecnologia e progresso tecnológico entre os agentes dos diferentes países. Nesse sentido, desde 1984, existe o Programa Ibero-americano de Ciência y Tecnología para el Desarrollo - Programa CYTED, criado para promover a cooperação entre os agentes de cada país membro deste programa, bem como criar e aperfeiçoar as competências tecnológicas dos mesmos. Para tanto, a configuração deste programa esta baseada em duas partes, uma primeira voltada para o desenvolvimento científico e tecnológico, e uma segunda vertente voltada para as empresas, neste caso, o Programa IBEROEKA. Este programa está relacionado com a articulação para a promoção da inovação tecnológica, através do fomento de projetos cooperativos entre as empresas da região Ibero-americana, também sendo desejável a participação de institutos de pesquisa e universidades.

O objetivo deste artigo é verificar a importância relativa do Programa CYTED como instrumento de articulação dos agentes latino-americanos e de cooperação internacional em matéria de ciência, tecnologia e inovação para países da América Latina.

A metodologia deste trabalho será estudo de caso. Esta metodologia permitirá verificar a importância relativa dos resultados das ações do Programa CYTED para o desenvolvimento científico, tecnológico e inovativo dos agentes ibero-americanos. O 
trabalho está estruturado da seguinte forma: primeiramente, far-se-á o referencial teórico baseado no Triângulo de Sábato e Sistema Nacional de Inovação para a constituição e consolidação de competências tecnológicas das estruturas desses países. Na segunda parte, discutir-se-á o papel da cooperação internacional por intermédio do Programa CYTED. Na terceira parte, apresentar-se-á o quadro geral das ações de cooperação internacional do Programa CYTED, com destaque da configuração das ações do referido programa.

Interação entre os agentes: uma breve descrição referencial teórico

O desenvolvimento científico, tecnológico e inovativo tem sido fundamental para a promoção da competitividade de produtos e serviços a serem produzidos. Schumpeter (1961) dividiu o processo de desenvolvimento tecnológico em três partes:

a) A invenção, como resultado de um processo de descoberta de novos princípios, com potencial de comercialização, mas não necessariamente realizada;

b) Inovação, como processo de desenvolvimento de uma invenção, já com intuito de comercializá-la e obter ganhos econômicos;

c) A difusão, como expansão da inovação, na forma de outros produtos ou processos.

Para tanto, este desenvolvimento requer a constituição e consolidação de interface entre os agentes. A interatividade dos agentes está atrelada aos arranjos institucionais e à sistemática para a promoção do desenvolvimento econômico. List (1841) analisou que a elaboração de políticas nacionais contribui no desenvolvimento da indústria nacional nascente associado com a sua proteção, relacionados diretamente à aquisição, ao aprendizado e à criação de novas tecnologias.

Já Botana e Sábato (1968) elaboraram uma metodologia de análise denominada Triângulo de Sábato, que permite compreender as interações existentes entre o Estado, as universidades e as empresas, a partir de cada vértice do Triângulo, quando considera complementares as competências de cada um destes agentes, o que possibilita a produção e o fluxo constante de troca de conhecimentos e tecnologias entre os mesmos.

Neste sentido, os principais agentes indutores do processo inovativo são ${ }^{(1)}$ :

a) Universidades e Institutos de Pesquisas — estes são responsáveis pela criação de conhecimento, desenvolvimento de pesquisa básica e aplicada e a formação de recursos humanos a serem alocados em pesquisas, e também daqueles a serem utilizados pelos demais agentes do sistema;

b) Estado - este agente é o formulador de políticas públicas (industrial, científica e tecnológica e macroeconômica). Por meio de políticas públicas e seus instrumentos,

(1) Apesar de existir uma quantidade de outros agentes no processo inovativo tais como agentes financeiros, organizações não governamentais. A descrição dos principais agentes é simplificada, sendo que o detalhamento esta em Teruya (2004). 
pretende-se promover a transferência de tecnologia entre os agentes envolvidos, e a dinâmica inovativa de setores produtivos, conforme a necessidade específica. Além disso, este agente é responsável pela regulação do comportamento dos agentes participantes;

c) Empresa - este agente é considerado o locus da inovação, e demanda tecnologias, treinamentos de seu capital humano, novos investimentos em P\&D, bens de capital, marketing e logística.

A motivação da formulação desta metodologia de interatividade entre os agentes está relacionada ao contex to latino-americano, em que as atividades de inovação são restritas nas empresas. Dessa forma, a interação é um dos fatores determinantes para congregar as competências científicas e tecnológicas no esforço inovativo, o que proporciona o compartilhamento dos riscos associados ao desenvolvimento tecnológico. Ao mesmo tempo, neste tipo de análise está implícita a governança das relações entre os agentes no sentido de promover a maior eficiência das interações entre os agentes envolvidos.

A partir das discussões de List (1841), e Botana e Sábato (1968), surgem as discussões relacionadas ao conceito de Sistema Nacional de Inovação, por intermédio de Freeman (1987), Lundvall (1992) e Nelson (1993). O processo de inovação tecnológica é entendido como sistêmico, de forma planejada ou não, composto por diversos agentes atuando cooperativamente, com uma coordenação mínima entre os mesmos, e possuindo estas diferentes capacidades e diferentes funções.

As redes de pesquisa se formam neste contexto e têm como uma de suas principais características a conjugação e a complementação de capacidades para consecução de resultados, o que possibilita a otimização na utilização de insumos e evita a duplicação de esforços e, concomitantemente, permite o compartilhamento de riscos associados ao desenvolvimento científico, tecnológico e inovativo.

Plonski (1995) considera três tipos de relações que ocorrem neste triângulo:

a) intrarrelações, entre os componentes de cada vértice;

b) interrelações, entre pares de vértices;

c) extrarrelações, as que se criam entre uma sociedade e o exterior.

Assim, Plonski (1995) considera o caráter fundamental das interrelações de tipo horizontal, entre a infraestrutura científico-tecnológica e a estrutura produtiva, sendo estas relações aquelas de mais difícil estabelecimento, e as mais interessantes de serem exploradas.

Ao mesmo tempo, para o desenvolvimento de novas tecnologias, é necessário o aprendizado tecnológico dos agentes envolvidos na dinâmica inovativa. A capacitação dos agentes envolvidos é importante para o desenvolvimento científico e tecnológico e da recepção da tecnologia desenvolvida por agentes nacionais ou de outros países (LUNDVALL et al., 2002). 
Associado a isto, as interações entre os agentes implicam na capacidade de apropriabilidade de tecnologia, que perpassa por elementos formais de interação, tais como o contrato de transferência de tecnologia, licenciamento de propriedade intelectual e convênios entre os agentes. Além disso, existem os mecanismos informais de transmissão da tecnologia, como a difusão às comunidades isoladas por intermédio de cursos para fim de conscientização e promoção do desenvolvimento local.

O desafio do processo inovativo para as estruturas latino-americanas é o estabelecimento de relações consistentes, de médio e longo prazo, entre os agentes. As instituições desses países apresentam deficiências, apresentando pouca capacidade para intermediar as relações entre os agentes, bem como a constituição de políticas consistentes e convergentes com o desenvolvimento econômico.

Uma das soluções para estas organizações e instituições é atuar cooperativamente através de redes internacionais, as quais congregam empresas e/ou universidades de diferentes países. Nesta análise das interfaces entre agentes nacionais e agentes internacionais de países ibero-americanos, parte-se do pressuposto que os agentes de diferentes países atuam em conformidade com os diferentes estágios de desenvolvimento científico e tecnológico de seus países, refletindo diversos graus de demandas tecnológicas. Por este motivo, é necessário considerar estas assimetrias para a realização destas interfaces, quando observadas num contexto da cooperação internacional. Ressalte-se que o sucesso das interações nas redes internacionais depende do estabelecimento de relações positivas entre seus componentes, os quais passam a desenvolver, cooperativamente, novas tecnologias. Estas devem constituir-se em resultados apropriáveis pela indústria, tornando-se passíveis de serem protegidos e difundidos para a sociedade.

\section{COOPERAÇÃo INTERNACIONAL EM CIÊNCIA, TECNOLOGIA E INOVAÇÃo PARA OS PAÍSES EM DESENVOLVIMENTO}

A cooperação internacional em matéria de ciência, tecnologia e inovação é um instrumento utilizado pelos agentes como forma de promover a capacitação em campos tecnológicos. Dessa forma, este instrumento não pode ser encarado como desacoplamento da autonomia da política nacional, mas sim um complemento à política nacional (COSTA FILHO, 2006).

Esta complementaridade está associada à complexidade do desenvolvimento científico, tecnológico e inovativo, bem como a falta de recursos financeiros para o investimento constante em Ciência e Tecnologia - C\&T - e Pesquisa e Desenvolvimento - P\&D. A continuidade dos investimentos é requerida, uma vez que os projetos aprovados são de longa duração e os resultados são compartilhados entre as partes envolvidas, quer seja em termos de publicação científica, quer seja no depósito de direito de propriedade intelectual, quer seja na repartição dos benefícios financeiros decorrentes desta ação conjunta.

Como há riscos associados ao processo tecnológico e inovativo, e incertezas nos resultados dos investimentos em $\mathrm{P} \& \mathrm{D}$, a formação de joint ventures entre os diferentes 
agentes é uma estratégia de compartilhamento de riscos e recursos humanos, capital e tecnologia. Tal estratégia, permite uma tentativa de otimização dos recursos envolvidos para o desenvolvimento tecnológico.

Sob as finalidades da cooperação internacional, tem-se a seguinte classificação (COSTA FILHO, 2006):

a) cooperação técnica — esta cooperação está mais próxima de uma consultoria e assistência técnica, sem uma interação efetiva de um dos lados. A interação é unidirecional na tentativa de absorção e transferência de tecnologia da parte receptora;

b) cooperação científica e técnica - esta cooperação envolve o desenvolvimento de tecnologia e/ou intercâmbio de conhecimento entre os agentes de diferentes países, o que poderá resultar na criação de competências tecnológicas locais e no desenvolvimento econômico das partes envolvidas;

c) cooperação financeira — esta cooperação está associada à operacionalização dos acordos assinados entre as partes envolvidas, ou seja, o financiamento das ativi-dades científicas e tecnológicas para a constituição e consolidação de infraestrutura para as atividades de C\&T e P\&D, tais como instalação, aquisição de equipamentos e capacitação de recursos humanos.

A relação entre os agentes de países desenvolvidos e em desenvolvimento, ou mesmo entre os países em desenvolvimentos de grau diferenciado de desenvolvimento científico e tecnológico, como é o caso da América Latina, podem ser assimétricas. A fragilidade de ganhos advindos de uma interação com agentes de diferentes países de sistema de inovação em países em desenvolvimento está relacionada com sua capacidade institucional limitada, o que compromete a capacidade de aprendizado e cumulatividade do conhecimento da parte menos estruturada.

Além disso, o aporte financeiro pelas partes envolvidas, a natureza da cooperação internacional, a criação de espaços interinstitucionais e as condições da infraestrutura dispo-nível das partes envolvidas influenciam a efetividade e a apropriação dos resultados da cooperação internacional.

Dessa forma, a cooperação internacional em Ciência, Tecnologia e Inovação - C\&T\&I pode não ocorrer de forma igualitária. Caso haja uma desigualdade na oferta entre as partes envolvidas, provavelmente não ocorrerá uma distribuição igualitária de funções e dos resultados advindos do processo de cooperação internacional. Além disso, os acordos de cooperação internacional, sua negociação e sua operacionalização são referências para evitar os constrangimentos das partes envolvidas e antever os possíveis ganhos advindos dos investimentos realizados da tecnologia desenvolvida.

A cooperação internacional pode ser um mecanismo de catching up para os agentes localizados em estruturas menos favorecidas, o que possibilitaria sua capacitação. No entanto, a geração de catching up está atrelada à mudança da configuração institucional, já que a absorção dos resultados alcançados pela cooperação e o avanço tecnológico demandariam uma evolução da estrutura relacional dos agentes. 
Os benefícios advindos de um processo de cooperação internacional são (COSTA FILHO, 2006):

a) Benefícios diretos - o compartilhamento dos riscos entre as partes envolvidas. O desenvolvimento científico, tecnológico e inovativo está associado ao risco. Assim, a formatação e o escopo delineado de projeto diminui os riscos, bem como os parceiros envolvidos têm suas funções definidas no projeto, partindo de suas competências tecnológicas já constituídas;

b) Benefícios indiretos - geração de grupos de pesquisas empresariais em determinados campos científicos e tecnológicos, que podem servir de referência regional e nacional.

Dessa forma, os países ibero-americanos podem ter benefícios diferenciados pelos programas de cooperação internacional em matéria de ciência, tecnologia e inovação, uma vez que o quadro de uma grande parte dos países ibero-americanos (sobretudo os países latino-americanos) apresentam um arranjo desarticulado dos agentes, uma estrutura deficiente de C\&T\&I, uma participação relativa baixa no comércio internacional e restrições nos indicadores macroeconômicos ${ }^{(2)}$.

Na próxima sessão, discutir-se-á a configuração e as ações do "Programa Ciencia y Tecnología para el Desarrollo” (Programa CYTED) para estimular o desenvolvimento científico, tecnológico e inovativo nos países da região Ibero-americana.

\section{Programa CYTED: configuração E aÇões de CiênCIA, TeCnologia e inovação}

A cooperação internacional em C\&T\&I tem sido corrente nos países da América Latina por conta do gap tecnológico e por conta do volume de investimentos contínuos em C\&T e P\&D necessários para o desenvolvimento tecnológico e inovativo. Dessa forma, articulações entre agentes de países da América Latina e de países desenvolvidos e ibéricos (Portugal e Espanha), e entre os agentes de países da América Latina, ocorreram como forma de atenuar os gargalos dentro dos Sistemas Nacionais de Inovação de cada país.

No contexto latino-americano, de um modo geral, as articulações e os investimentos em C\&T e P\&D não estão estruturados. Em contrapartida, existe uma diferença de estrutura institucional entre os países da América Latina, e isto está relacionado com o nível de investimento de cada país e a capacidade de desenvolvimento tecnológico endógeno. Pode ser considerado que Argentina, Brasil, México e Chile estão na vanguarda latino-americana. Estes países já apresentam programas de desenvolvimento tecnológico e uma estrutura mínima com uma propensão a inovar. Enquanto isto, Bolívia, Paraguai, El Salvador, e Honduras, entre outros, encontram-se em uma situação caracterizada pela baixa capacidade de organização interna, das interações entre os agentes relacionados a C\&T e P\&D.

\footnotetext{
(2) Ressalva-se que cada país da região ibero-americana se encontra em estágios diferenciados de articulação entre os agentes e do grau de geração e apropriação da tecnologia gerada. No entanto, os países apresentam debilidades, que restringem o progresso tecnológico.
} 
Assim, o Programa CYTED foi criado em 1984, a partir de um Acordo Marco entre os países ibero-americanos, e com as finalidades de promover o desenvolvimento científico e tecnológico dos agentes dos países membros, promover a integração regional em termos de ciência, tecnologia e inovação, estimular a participação das firmas dos países membros interessados na atividade inovativa e estimular a participação de pesquisadores em outros programas multilaterais (HIDALGO NICHERA; GARRIGÓS, 2004).

Este programa representa uma tentativa de promover a cooperação internacional entre universidades, institutos de pesquisas e empresas da região Ibero-americana. $\mathrm{O}$ Programa CYTED ${ }^{(3)}$ apresenta a seguinte configuração:

a) Cooperação multilateral — o programa apresenta 21 países membros, sendo que cada país apresenta o mesmo peso na tomada de decisão, conforme o Quadro 1;

b) Programa horizontal — esta característica está relacionada com o pressuposto de que as responsabilidades e os benefícios são compartilhados com os países membros;

c) Programa descentralizado — os participantes têm poder de influência relativa nas decisões das diferentes instâncias do programa;

d) Focalização do programa — o escopo do programa está centrado no desenvolvimento científico, tecnológico e inovativo, por meio dos instrumentos disponíveis pelo Programa;

e) Configuração em rede - o programa está baseado na constituição de atividades científicas, tecnológicas e inovativas em redes.

\section{Quadro 1 - Países membros do Programa CYTED e seus respectivos Organismos Signatários e Organismo Gestor IBEROEKA ${ }^{(4)}$}

\begin{tabular}{|c|l|l|}
\hline País & \multicolumn{1}{|c|}{ Organismo Signatário } & \multicolumn{1}{c|}{ Organismo Gestor IBEROEKA } \\
\hline Argentina & $\begin{array}{l}\text { Ministerio de Ciencia, Tecnología e } \\
\text { Innovación Productiva }\end{array}$ & $\begin{array}{l}\text { Ministerio de Ciencia, Tecnología } \\
\text { e Innovación Productiva }\end{array}$ \\
\hline \multirow{2}{*}{ Bolívia } & $\begin{array}{l}\text { Viceministerio de Ciencia y } \\
\text { Tecnología, Ministerio de } \\
\text { Planificación del Desarrollo }\end{array}$ & $\begin{array}{l}\text { Viceministerio de Ciencia y } \\
\text { Tecnología, Ministerio de } \\
\text { Planificación del Desarrollo }\end{array}$ \\
\hline
\end{tabular}

(3) A estrutura administrativa do Programa CYTED está baseada em uma Secretaria Geral sediada na Espanha e duas Secretarias Adjuntas localizadas na América Central e a outra na América do Sul. Estas possuem, entre suas funções, a função de prover suporte administrativo e realização de um trabalho de prospecção com os agentes nacionais responsáveis pelas atividades de inovação científica e tecnológica.

(4) Os organismos signatários do Programa CYTED na parte científica e tecnológica podem ser diferentes em relação ao organismo Gestor Iberoeka, pois o foco de cada vertente é diferente. Neste caso, o Brasil apresenta como Organismo Gestor IBEROEKA, a Financiadora de Estudos e Projetos — FINEP e organismo signatário é o Conselho Nacional de Desenvolvimento Científico e Tecnológico - CNPq. Alguns países apresentam a mesma organização, que atua tanto como Organismo Gestor IBEROEKA quanto Organismo Signatário. 


\begin{tabular}{|c|c|c|}
\hline País & Organismo Signatário & Organismo Gestor IBEROEKA \\
\hline Brasil & $\begin{array}{l}\text { Conselho Nacional de } \\
\text { Desenvolvimento Científico e } \\
\text { Tecnológico }\end{array}$ & $\begin{array}{l}\text { Financiadora de Estudos e } \\
\text { Projetos }\end{array}$ \\
\hline Chile & $\begin{array}{l}\text { Comisión Nacional de Investigación } \\
\text { Científica y Tecnológica }\end{array}$ & $\begin{array}{l}\text { Fondo de Fomento al Desarrollo } \\
\text { Científico y Tecnológico - } \\
\text { Comisión Nacional de Investi- } \\
\text { gación Científica y Tecnológica }\end{array}$ \\
\hline Colômbia & $\begin{array}{l}\text { Departamento Administrativo de } \\
\text { Ciencia, Tecnología e Innovación }\end{array}$ & $\begin{array}{l}\text { Departamento Administrativo de } \\
\text { Ciencia, Tecnología e Innovación }\end{array}$ \\
\hline Costa Rica & Ministerio de Ciencia y Tecnología & $\begin{array}{l}\text { Ministerio de Ciencia y } \\
\text { Tecnología }\end{array}$ \\
\hline Cuba & $\begin{array}{l}\text { Ministerio de Ciencia, Tecnología y } \\
\text { Medio Ambiente }\end{array}$ & $\begin{array}{l}\text { Ministerio para la Inversión } \\
\text { Extranjera y la Colaboración } \\
\text { Económica }\end{array}$ \\
\hline El Salvador & $\begin{array}{l}\text { Consejo Nacional de Ciencia y } \\
\text { Tecnología }\end{array}$ & $\begin{array}{l}\text { Consejo Nacional de Ciencia y } \\
\text { Tecnología }\end{array}$ \\
\hline Equador & $\begin{array}{l}\text { Secretaría Nacional de Ciencia y } \\
\text { Tecnología }\end{array}$ & $\begin{array}{l}\text { Secretaría Nacional de Ciencia y } \\
\text { Tecnología }\end{array}$ \\
\hline Espanha & $\begin{array}{l}\text { Agencia Española de Cooperación } \\
\text { Internacional para el Desarrollo / } \\
\text { Ministerio de Ciencia e Innovación }\end{array}$ & $\begin{array}{l}\text { Centro para el Desarrollo } \\
\text { Tecnológico Industrial }\end{array}$ \\
\hline Guatemala & $\begin{array}{l}\text { Consejo Nacional de Ciencia y } \\
\text { Tecnología / Secretaría Nacional de } \\
\text { Ciencia y Tecnología }\end{array}$ & $\begin{array}{l}\text { Consejo Nacional de Ciencia y } \\
\text { Tecnología / Secretaría Nacional } \\
\text { de Ciencia y Tecnología }\end{array}$ \\
\hline Honduras & $\begin{array}{l}\text { Consejo Hondureño de Ciencia y } \\
\text { Tecnología }\end{array}$ & $\begin{array}{l}\text { Consejo Hondureño de Ciencia y } \\
\text { Tecnología }\end{array}$ \\
\hline México & $\begin{array}{l}\text { Consejo Nacional de Ciencia y } \\
\text { Tecnología }\end{array}$ & $\begin{array}{l}\text { Consejo Nacional de Ciencia y } \\
\text { Tecnología }\end{array}$ \\
\hline Nicarágua & $\begin{array}{l}\text { Consejo Nicaragüense de Ciencia y } \\
\text { Tecnología }\end{array}$ & $\begin{array}{l}\text { Consejo Nicaragüense de Ciencia } \\
\text { y Tecnología }\end{array}$ \\
\hline Panamá & $\begin{array}{l}\text { Secretaría Nacional de Ciencia, } \\
\text { Tecnología e Innovación }\end{array}$ & $\begin{array}{l}\text { Secretaría Nacional de Ciencia, } \\
\text { Tecnología e Innovación }\end{array}$ \\
\hline Paraguai & $\begin{array}{l}\text { Instituto Nacional de Tecnología, } \\
\text { Normalización y Metrología }\end{array}$ & $\begin{array}{l}\text { Instituto Nacional de Tecnología, } \\
\text { Normalización y Metrología }\end{array}$ \\
\hline Peru & $\begin{array}{l}\text { Consejo Nacional de Ciencia, } \\
\text { Tecnología e Innovación Tecnológica }\end{array}$ & $\begin{array}{l}\text { Consejo Nacional de Ciencia, } \\
\text { Tecnología e Innovación } \\
\text { Tecnológica }\end{array}$ \\
\hline Portugal & $\begin{array}{l}\text { Ministério da Ciência, Tecnologia e } \\
\text { Ensino Superior }\end{array}$ & Agência de Inovação \\
\hline
\end{tabular}




\begin{tabular}{|c|l|l|}
\hline País & \multicolumn{1}{|c|}{ Organismo Signatário } & \multicolumn{1}{|c|}{ Organismo Gestor IBEROEKA } \\
\hline $\begin{array}{c}\text { República } \\
\text { Dominicana }\end{array}$ & $\begin{array}{l}\text { Secretaría de Estado de Educación } \\
\text { Superior, Ciencia y Tecnología }\end{array}$ & $\begin{array}{l}\text { Instituto de Innovación en } \\
\text { Biotecnología e Industria }\end{array}$ \\
\hline Uruguai & Ministerio de Educación y Cultura & $\begin{array}{l}\text { Agencia Nacional de } \\
\text { Investigación e Innovación }\end{array}$ \\
\hline Venezuela & $\begin{array}{l}\text { Ministerio del Poder Popular para } \\
\text { Ciencia, Tecnología e Industrias } \\
\text { Intermedias }\end{array}$ & $\begin{array}{l}\text { Ministerio del Poder Popular para } \\
\text { Ciencia, Tecnología e Industrias } \\
\text { Intermedias }\end{array}$ \\
\hline
\end{tabular}

Fonte: Programa CYTED.

A estrutura de governança do Programa CYTED está baseada na participação dos países signatários nas instâncias máximas do Programa, que são o Conselho Diretivo ${ }^{(5)}$ e a Assembleia Geral ${ }^{(6)}$. Esta configuração do Programa CYTED permite a participação dos diversos agentes dos países membros ao programa, sendo que os representantes signatários têm a capacidade de sugerir e ratificar as decisões tomadas no âmbito do programa.

O Programa CYTED apresenta duas vertentes básicas de atuação, a primeira é voltada para a parte científica e tecnológica, e a segunda, direcionada para projetos de inovação.

A vertente científica e tecnológica está organizada em Áreas Temáticas ${ }^{(7)}$. Atualmente, esta parte apresenta uma estrutura de gestão, o Comitê Gestor de Área Temática, que delineiam e avaliam as propostas submetidas ao programa. Esta área do Programa CYTED recebe aporte financeiro dos países signatários ao Programa para subvenção das atividades de pesquisas e de disseminação do conhecimento gerado.

Em relação ao orçamento destinado às atividades de financiamento de Ciência e Tecnologia, observa-se que houve um aumento dos recursos empregados entre 1984 a 1997. Contudo, houve um ponto de inflexão com a queda entre 1997 e 1998, sendo que, a partir de 1998, houve um novo incremento, mas não superou o aporte de 1997, em termos correntes, conforme o Gráfico 1.

Cabe destacar que existe uma desigualdade de participação financeira dos países membros ao Programa CYTED ${ }^{(8)}$. O maior contribuinte ao Programa CYTED tem sido a

(5) O Conselho Diretivo do Programa CYTED é responsável pela recomendação à Assembléia Geral o orçamento anual, aprovação da distribuição do orçamento por unidades de ação, avaliação das áreas temáticas do Programa, proposição de linhas temáticas a serem criadas pelas áreas temáticas e aprovação da transferência de tecnologia.

(6) A Assembleia Geral tem a aprovação do orçamento e sua distribuição pelas propostas apresentadas, aprovação dos informes de gestão e avaliação elaborados, respectivamente, pela Secretaria Geral e pelo Conselho Diretivo, aprovação dos novos gestores de áreas temáticas e aprovação de novos convênios.

(7) Cada Área Temática apresenta um Gestor de área e os membros que são nomeados pela Assembleia Geral do Programa CYTED. Atualmente, existem sete áreas temáticas: Agroalimentação; Saúde; Promoção de Desenvolvimento Industrial; Desenvolvimento Sustentável, Mudanças Globais e Ecossistemas; Tecnologia das Comunicações e Informação; Ciência e Sociedade; Energia.

(8) Ressalta-se que cada país membro tem condições iguais de decisão nas reuniões do Conselho Diretivo e da Assembleia Geral. 
Espanha, responsável por quase 75\% dos recursos financeiros destinados à parte científica e tecnológica e administrativa. Percebe-se pela, Tabela 1, que os maiores contribuintes ao Programa, em 2006, são Espanha, Brasil, México, Portugal, Venezuela, Chile e Argentina, que apresentam uma estrutura de C\&T e P\&D mais estruturado na região Ibero-americana. Esta situação, também, é decorrente da capacidade econômica dos países e da relevância do Programa CYTED para estes países, em relação a suas políticas nacionais dos países membros.

\section{Gráfico 1 - Orçamento do Programa CYTEC entre 1984 a 2003 em US $\$$ correntes}

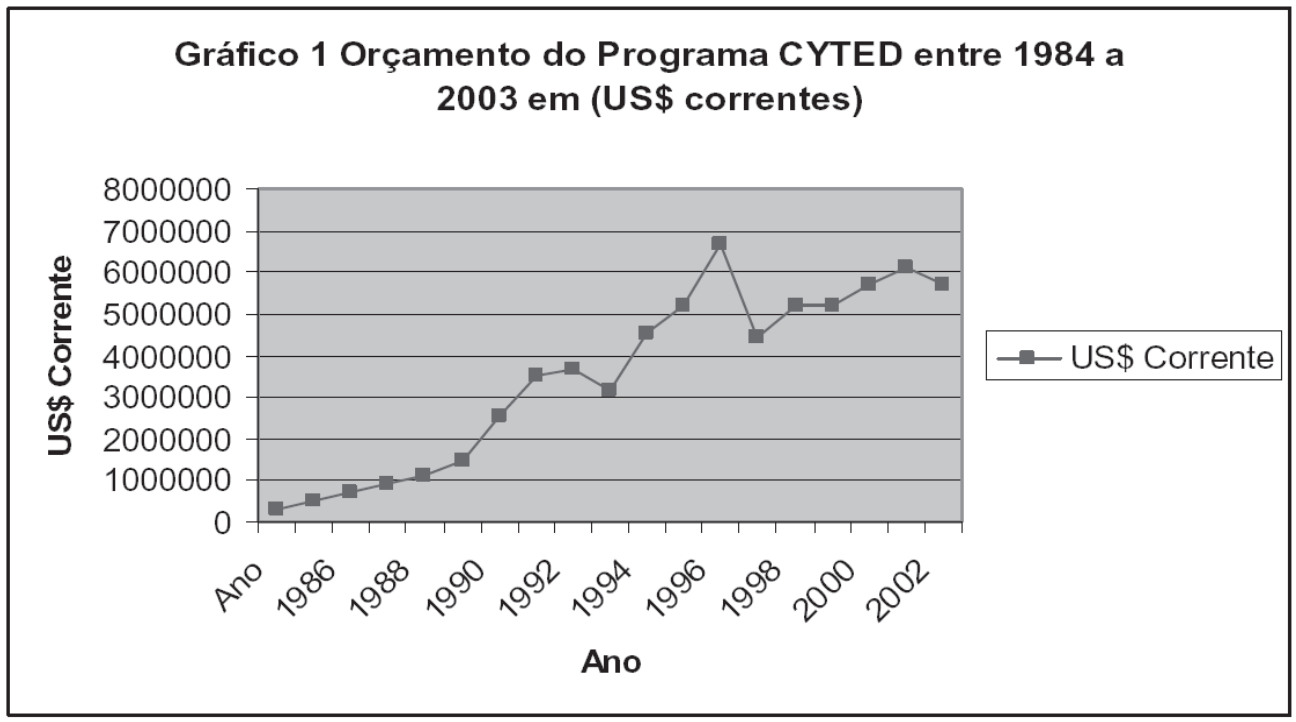

Fonte: Programa CYTED.

Por sua vez, um grupo de países latino-americanos, membros do Programa CYTED, apresentam contribuição financeira pouco significativa ao Programa, por conta de suas limitações de recursos financeiros dedicados a apoiar C\&T e P\&D. Desta forma, este programa apresenta um peso relativamente importante dentro da estrutura fragilizada de C\&T e P\&D destes países, como, por exemplo, El Salvador, Nicarágua, Honduras, Guatemala, República Dominicana e Paraguai, conforme exposto na Tabela 1.

Esta diferença no peso relativo das contribuições ao Programa tem impactos diferenciados nos países membros. Aqueles países que contribuem pouco financeiramente e não apresentam um Sistema Nacional de Inovação organizado, como os casos dos países da América Central e alguns países da América do Sul, o Programa CYTED tem um impacto grande nas estruturas de ciência e tecnologia, pois possibilita a difusão do conhecimento gerado pela cooperação internacional, nas modalidades apoiadas pelo Programa CYTED, a estes países. Já os países com maior contribuição possuem outros programas nacionais de envergadura horizontal e, devido a este fato, o Programa CYTED apresenta uma importância relativa menor, se comparado com os países da América Central. 
Tabela 1 - Participação na contribuição ao Programa CYTED pelos países signatários em 2006

\begin{tabular}{|c|c|c|}
\hline País & Participação em \% & Direta/Indireta \\
\hline Espanha & 74,18 & Direta \\
\hline Brasil & 7,42 & Indireta \\
\hline México & 4,75 & Direta \\
\hline Portugal & 3,71 & Direta \\
\hline Venezuela & 2,37 & Direta \\
\hline Chile & 1,71 & Indireta \\
\hline Argentina & 1,48 & Direta \\
\hline Cuba & 0,74 & Direta \\
\hline Colômbia & 0,52 & Direta \\
\hline Peru & 0,52 & Direta \\
\hline Costa Rica & 0,37 & Indireta \\
\hline República Dominicana & 0,37 & Direta \\
\hline Uruguai & 0,37 & Indireta \\
\hline Panamá & 0,30 & Direta \\
\hline Bolívia & 0,22 & Direta \\
\hline Equador & 0,22 & Direta \\
\hline El Salvador & 0,15 & Indireta \\
\hline Guatemala & 0,15 & Indireta \\
\hline Honduras & 0,15 & Direta \\
\hline Nicarágua & 0,15 & Indireta \\
\hline Paraguai & 0,15 & Indireta \\
\hline TOTAL & $100,0 \%$ & \\
\hline
\end{tabular}

Fonte: Programa CYTED.

Um outro aspecto é a forma da contribuição financeira dos organismos signatários. Uma parte dos membros faz sua contribuição de forma indireta, ou seja, eles repassam os recursos diretamente aos coordenadores de projetos ou a membros dos projetos de seus países. Como consequência, tem-se uma duplicação de esforços, uma vez que existe uma duplicação de esforços na gestão financeira pela Secretária Geral do Programa CYTED, dos recursos direcionados às propostas aprovadas, é necessário que esta Secretaria controle tanto os recursos advindos diretamente pelos membros signatários quanto aqueles recursos que são repassados diretamente aos coordenadores de projetos ou a membros de projetos. 
Estes recursos enviados diretamente ao Programa CYTED são utilizados na parte administrativa do Programa e nos instrumentos da parte de C\&T gerenciada pelas áreas temáticas do Programa CYTED. Os instrumentos são(9):

a) Redes Temáticas — as Redes Temáticas são definidas como associações de grupos de pesquisa consolidados de países participantes no Programa CYTED, que tenham interesse comum e pratiquem atividades numa mesma área de conhecimento. Estas redes têm como objetivo principal facilitar as relações e a difusão de conhecimento entre os grupos de pesquisa, formação de recursos humanos, capacitação técnica e metodológica, ação de promoção e transferência de tecnologia aos demais agentes, em âmbito ibero-americano;

b) Ações de Coordenação de Projetos de Pesquisa — as ações de Coordenação visam a mobilidade de pesquisadores, que trabalham em um projeto de pesquisa comum dos agentes públicos e privados da região Ibero-americana. Também, apresenta como objetivo a transferência de tecnologia para as empresas;

c) Projetos de Pesquisa Consorciados — essa linha de financiamento visa o desenvolvimento e/ou aprimoramento de produtos, processos, e serviços, a partir das competências dos agentes envolvidos e que produza ganhos econômicos aos agentes. Diferente das modalidades anteriores, esta linha de financiamento possibilita a aquisição de bens de capital para o desenvolvimento do projeto aprovado.

As propostas apoiadas pelos instrumentos do Programa CYTED podem ter outras fontes de financiamento para o desenvolvimento de suas atividades. Com isto, pode haver uma complementaridade de fundos nacionais, aperfeiçoando as sinergias com os agentes de cada país membro. Assim, as propostas submetidas e aprovadas, necessariamente, precisam convergir com as demandas nacionais. Além disso, a participação de agentes públicos e privados de diferentes países membros contribui para a constituição de competências tecnológicas que podem ser compartilhadas entre os países.

O processo de difusão da tecnologia é uma etapa importante para a capacitação e formação de recursos humanos, tanto de alta qualificação quanto para as comunidades locais da região Ibero-americanana. Ao mesmo tempo, este conhecimento de tecnologias é transformado em riqueza econômica e gera inovação tecnológica, o que aumenta a importância de projetos de promoção de inovação tecnológica nas firmas ibero-americanas por meio de cooperação internacional.

Nesse sentido, conforme a Tabela 2, as propostas em vigência no Programa CYTED estão concentradas nas sete áreas temáticas. Dentre as modalidades de fomento deste programa, destaca uma alta participação das redes temáticas, que representam quase $68 \%$ das propostas vigentes. Isso se deve ao fato de que as redes temáticas são atividades de

(9) As propostas aprovadas são decorrentes daquelas submetidas a editais públicos do Programa CYTED, sendo avaliadas por consultores ad hoc e pelos Comitês Gestores de cada área Temática, sendo as decisões ratificadas no Conselho Diretivo e na Assembleia Geral. 
pesquisa de cada grupo de pesquisas consolidadas na região Ibero-americana, com a coordenação dos trabalhos estruturados na forma de rede, com uma temática em comum. Convém destacar que as áreas temáticas de Saúde e Desenvolvimento Sustentável, Mudanças Climáticas e Ecossistemas com maior número de redes em vigência.

Já, os Projetos de Pesquisas Consorciados apresentam uma participação relativa muito baixa (com quatro projetos em andamento), pois apresenta recursos financeiros da ordem de US $\$ 200$ mil por ano de projeto, já que tem a pretensão do desenvolvimento de protótipos e é de aplicação industrial, o que requer um investimento substancialmente mais elevado. Além destas, há também as Ações de Coordenação, que representam, aproximadamente, $31 \%$ das ações financiadas em vigência. As áreas temáticas de Promoção de Desenvolvimento Industrial, Desenvolvimento Sustentável, Mudanças Climáticas e Ecossistemas, Energia e Tecnologias das Comunicações e Informações são as que mais têm projetos em vigência com 8 e 6 projetos, respectivamente.

Assim, na participação relativa dos projetos em vigência por área temática na totalidade das modalidades aprovadas, percebe-se um equilíbrio de propostas financiadas pelo Programa CYTED, sendo que, 20\% dos projetos estão na área de Desenvolvimento Sustentável, Mudanças Climáticas e Ecossistemas; $16 \%$ dos projetos são da área de Saúde; 15,2\% são das áreas de Agroalimentação e Promoção do Desenvolvimento Industrial; 12\% dos projetos estão alocados na Ciência e Sociedade; e 8,8\% dos projetos são da área de Energia ${ }^{(10)}$.

\section{Tabela 2 - Número de redes de pesquisas, ações de coordenação, projetos de pesquisas consorciados iniciadas entre 2007 e 2010, e em vigência, por áreas temáticas}

\begin{tabular}{|l|c|c|c|}
\hline \multicolumn{1}{|c|}{ Área Temática } & $\begin{array}{c}\text { Redes } \\
\text { Temáticas }\end{array}$ & $\begin{array}{c}\text { Ações de } \\
\text { Coordenação }\end{array}$ & $\begin{array}{c}\text { Projetos de } \\
\text { Pesquisas } \\
\text { Consorciadas }\end{array}$ \\
\hline Agroalimentação & 11 & 04 & 1 \\
\hline Saúde & 18 & 00 & 0 \\
\hline Promoção do Desenvolvimento Industrial & 07 & 08 & 0 \\
\hline $\begin{array}{l}\text { Desenvolvimento sustentável, Mudanças } \\
\text { Climáticas e Ecossistemas }\end{array}$ & 11 & 06 & 0 \\
\hline Tecnologias das Comunicações e Informações & 06 & 06 & 0 \\
\hline Ciência e Sociedade & 13 & 01 & 1 \\
\hline Energia & 02 & 06 & 3 \\
\hline Total & 68 & 31 & 1 \\
\hline
\end{tabular}

Fonte: Programa CYTED.

(10) Esta área de energia é nova no Programa CYTED, que se iniciou em 2007, por isso o número de projetos financiados ainda é baixo, se comparado com as outras áreas temáticas. 
Cada Rede temática, Ação de Coordenação e Projeto de Pesquisa Consorciada financiado pelo programa CYTED apresenta um coordenador geral proveniente de uma instituição pública ou privada de cada um dos países membros do programa. Cada proposta aprovada congrega grupos de pesquisas e/ou empresas de diferentes países iberoamericanos, no mínimo de quatro países diferentes da Região Ibero-americana.

A seleção das propostas leva em conta a pertinência da temática a ser estudada, o número de países envolvidos e de grupos de pesquisas consolidados e emergentes (originados de países com pouca estruturação de seu Sistema Nacional de Inovação) participantes, e da presença de empresas, bem como a capacidade de difusão do conhecimento gerado para a sociedade por intermédio de jornadas, cursos e seminários.

Dessas propostas em vigência, apresenta-se uma maioria de Ações de Coordenação e Redes Temáticas coordenadas por espanhóis, com uma participação de 51,72\% e 33,82\%, respectivamente. Verifica-se que a Espanha, como maior contribuinte, tem maior participação tanto na coordenação quanto destaque na participação dos grupos de pesquisas nas Ações de Coordenação e Redes Temáticas, conforme a Tabela 3.

Também, na Tabela 3, verifica-se que países como Argentina, Cuba, México e Portugal atuam na coordenação de Redes Temáticas e Ações de Coordenação vigentes, tendo, ainda, uma participação significativa nas redes temáticas e ações de coordenação. No caso brasileiro, tem-se uma grande participação nas redes e ações de coordenação vigentes, mas pouca participação na coordenação de redes temáticas, ainda que apresente a segunda maior participação relativa na coordenação de Ações de Coordenação, com cerca de 13,8\%.

Observa-se também que não há, atualmente, coordenadores oriundos de países como Peru, Panamá, Nicarágua, El Salvador, Honduras, Guatemala e Paraguai, tanto nas Ações de Coordenação quanto nas Redes Temáticas, ainda que estes países participem em redes e ações de coordenação, e mesmo que sua participação relativa seja menor se comparado com Sistemas Nacionais de Inovação mais estruturados. Cabe destacar que esta participação de grupos de pesquisas destes países possibilita a interação com outros grupos de pesquisas ibero-americanos, proporcionando um aprendizado científico e tecnológico, bem como a possibilidade de participação de jornadas científicas e qualificação dos recursos humanos locais.

Em relação aos Projetos de Pesquisas Consorciados, há três projetos vigentes com participação de agentes de poucos países. Isto se deve à envergadura e ao escopo de projeto, uma vez que estes devem englobar a produção de unidades piloto, voltadas para satisfazer a demandas do setor empresarial ibero-americano. 
Tabela 3 - Participação dos países signatários do Programa CYTED nos projetos vigentes 2006 a 2010

\begin{tabular}{|c|c|c|c|c|c|c|}
\hline \multirow[b]{2}{*}{ País } & \multicolumn{2}{|c|}{ Redes Temáticas } & \multicolumn{2}{|c|}{$\begin{array}{c}\text { Ações de } \\
\text { Coordenação }\end{array}$} & \multicolumn{2}{|c|}{$\begin{array}{c}\text { Projeto de Pesquisa } \\
\text { Consorciado }\end{array}$} \\
\hline & Coordenador & Membros & Coordenador & Membros & Coordenador & Membros \\
\hline Espanha & 33,82 & 82,35 & 51,72 & 86,21 & 66,67 & 66,67 \\
\hline Brasil & 5,88 & 80,88 & 13,79 & 86,21 & 0,00 & 0,00 \\
\hline México & 8,82 & 67,65 & 6,90 & 62,07 & 33,33 & 33,33 \\
\hline Portugal & 7,35 & 50,00 & 3,45 & 65,52 & 0,00 & 33,33 \\
\hline Venezuela & 1,47 & 44,12 & 0,00 & 41,38 & 0,00 & 0,00 \\
\hline Chile & 2,94 & 58,82 & 0,00 & 58,62 & 0,00 & 33,33 \\
\hline Argentina & 16,18 & 85,29 & 10,34 & 79,31 & 0,00 & 33,33 \\
\hline Cuba & 16,18 & 42,65 & 0,00 & 55,17 & 0,00 & 33,33 \\
\hline Colômbia & 2,94 & 48,53 & 3,45 & 44,83 & 0,00 & 66,67 \\
\hline Peru & 0,00 & 30,88 & 0,00 & 34,48 & 0,00 & 33,33 \\
\hline Costa Rica & 1,47 & 27,94 & 0,00 & 17,24 & 0,00 & 0,00 \\
\hline República Dominicana & 0,00 & 7,35 & 3,45 & 0,00 & 0,00 & 0,00 \\
\hline Uruguai & 2,94 & 33,82 & 3,45 & 37,93 & 0,00 & 33,33 \\
\hline Panamá & 0,00 & 10,29 & 0,00 & 6,90 & 0,00 & 0,00 \\
\hline Bolívia & 0,00 & 20,59 & 3,45 & 13,79 & 0,00 & 33,33 \\
\hline Equador & 0,00 & 36,76 & 0,00 & 20,69 & 0,00 & 33,33 \\
\hline El Salvador & 0,00 & 5,88 & 0,00 & 0,00 & 0,00 & 0,00 \\
\hline Guatemala & 0,00 & 16,18 & 0,00 & 6,90 & 0,00 & 0,00 \\
\hline Honduras & 0,00 & 10,29 & 0,00 & 3,45 & 0,00 & 33,33 \\
\hline Nicarágua & 0,00 & 13,24 & 0,00 & 6,90 & 0,00 & 0,00 \\
\hline Paraguai & 0,00 & 11,76 & 0,00 & 13,79 & 0,00 & 0,00 \\
\hline
\end{tabular}

Fonte: Programa CYTED.

Uma segunda vertente do Programa CYTED é o Programa IBEROEKA ${ }^{(1)}$, voltado para a atividade inovativa das firmas dos países membros do CYTED. Organizado de forma cooperada, este instrumento é gerenciado, em cada país membro, pelo Organismo Gestor IBEROEKA nacional, sob o auspício do Programa CYTED.

(11) O IBEROEKA foi inspirado no Programa EUREKA, da Comissão Européia, e é destinado a estimular as empresas dos países participantes do Programa CYTED, na busca de parcerias para o desenvolvimento de projetos cooperativos de cunho inovador. 
As atividades do IBEROEKA, exceto sua administração, não são financiadas pelo Programa CYTED. As empresas formam o consórcio de empresas e elaboram o projeto, no qual podem, eventualmente, participar de institutos de pesquisas. A partir da elaboração do projeto e da definição dos membros do consórcio, estes projetos são submetidos ao IBEROEKA, para certificação. Em caso de aprovação desta certificação, os membros do consórcio buscam fontes de financiamento para seus projetos, através de financiamentos dos Organismos Gestores nacionais, ou por outras fontes nacionais e internacionais públicas e privadas (HIDALGO NICHERA; GARRAGÓS, 2004).

Este instrumento de cooperação internacional apresenta uma importância relativa, segundo o Gráfico 2, o número de projetos certificados obteve um aumento entre 1991 a 2002, sendo que o número de projetos certificados foi de 66, em 2002. A partir de 2002, houve uma oscilação de projetos certificados, sendo, em 2008, o número de 46.

Como aspecto positivo deste instrumento do ponto de vista das empresas, destaca-se (HIDALGO NICHERA; GARRAGÓS, 2004):

a) inserção de produtos em novos mercados;

b) desenvolvimento conjunto de tecnologias com institutos de pesquisas, universidades e empresas, com isso, compartilha-se o risco do processo inovativo;

c) acesso a novos conhecimentos complementares às competências das empresas envolvidas;

d) possibilidade de acesso a fontes de financiamento público e privado para as atividades de P\&D;

e) possibilidade de acesso a novos mercados potenciais na região Ibero-americana.

\section{Gráfico 2 - Números de projetos certificados no Projeto Iberoeka e frequência acumulada de 1991 a 2008}

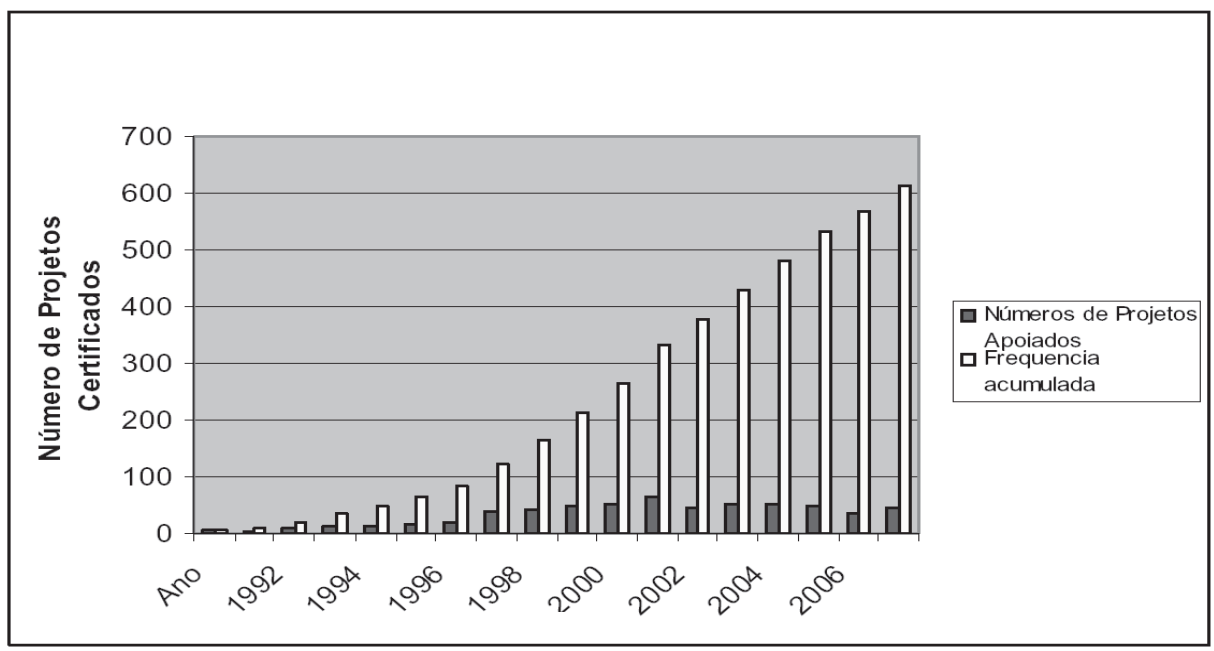

Fonte: Programa CYTED. 
Neste sentido, a formação de consórcios interempresariais e a participação de institutos de pesquisas e universidades da região ibero-americana é importante para a formação de competências tecnológicas, a geração de inovação tecnológica em produtos e processos, visando a formação de uma cadeia logística e de comercialização de bens e serviços na região.

Em contrapartida, existem aspectos limitantes deste instrumento como forma de promover o melhor desempenho do consórcio; cabe destacar os seguintes aspectos (HIDALGO NICHERA; GARRAGÓS, 2004):

a) crise econômica global tem diminuído as oportunidades de mercado, os investimentos de médio e longo prazo, incluindo os de P\&D;

b) problemas institucionais dos países ibero-americanos que limitam a difusão da inovação tecnológica;

c) dificuldade de coordenação das atividades de cada membro do consórcio.

d) falta de linhas de financiamentos específicos em muitos países da América Latina;

e) poucos mecanismos de articulação da política nacional de inovação tecnológica com projetos de internacionalização das firmas nos países ibero-americanos;

f) dificuldade no encontro de parceiros em outros países ibero-americanos.

Observa-se, ainda nesta região, uma baixa articulação entre universidade e empresas, sendo que uma parte da explicação está relacionada pela imaturidade das relações entre os vértices do Triângulo de Sábato. Além disso, a atividade inovativa nos países latino-americanos é pouco significativa, somente em alguns setores produtivos.

Tanto as ações científicas e tecnológicas quanto inovativas no âmbito do Programa CYTED não são condições suficientes para promover o catching up dos países ibero-americanos, pois ainda tem-se a restrição da limitação de articulação dos instrumentos nacionais como os internacionais, bem como a heterogeneidade entre os países membros do Programa CYTED. Adicionado a isso, os países ibero-americanos encontram-se com dificuldades institucionais para arquitetar uma articulação eficiente dos agentes envolvidos, bem como a resolução de problemas macroeconômicos de magnitudes globais.

Dessa forma, é complexo atender a diferentes demandas de cada país de um modo particular por meio do Programa CYTED, pois há, de um lado, países com Sistemas Nacionais de Inovação mais estruturados, como Espanha, Portugal, Brasil e México, enquanto há outros cujos Sistemas Nacionais ainda se encontram em fase de constituição, tais como Bolívia, El Salvador, Guatemala, Paraguai e República Dominicana. No entanto, este programa tem sido um marco importante na região Ibero-americana, como referência de cooperação internacional multilateral.

\section{Conclusão}

A interação entre os agentes é fundamental para o desenvolvimento científico, tecnológico e inovativo. Assim, quanto maior for a integração dos agentes, maior será a probabilidade de promoção da atividade inovativa. 
No entanto, em regiões onde há poucos instrumentos nacionais, como é o caso da América Latina, observa-se uma articulação limitada dos agentes por conta de uma institucionalidade limitada, a qual restringe o progresso tecnológico dos países. Acrescentese que há diferenças nas configurações institucionais, que permitem existir, em alguns países, uma estrutura científica, tecnológica e inovativa mais consolidada em relação aos outros. Mesmo assim, verifica-se-se um gap tecnológico em relação entre os países membros dos países ibero-americanos com os países da fronteira tecnológica.

O Programa CYTED é um instrumento aglutinador dos vértices dos diversos triângulos de Sábato dos países ibero-americanos, que apresentam diferentes níveis de desenvolvimento tecnológico e econômico. Entretanto, este instrumento de cooperação internacional, embora por si só não gere condições suficientes para promover articulações entre os agentes, é capaz de promover o desenvolvimento científico, tecnológico e inovativo conjunto, bem como a disseminação deste conhecimento gerado aos agentes locais.

O desafio é a conciliação de interesses diversos dos países membros do Programa CYTED, para que se possa promover externalidades positivas dinâmicas aos agentes envolvidos nos instrumentos do programa em um contexto de disparidade da configuração do Triângulo de Sábato, de cada país.

Da mesma forma, verifica-se que o mecanismo do IBEROEKA vem se apresentando como um instrumento válido de integração da vertente científica e tecnológica empresarial da região ibero-americana. No entanto, o IBEROEKA precisa estar conjugado com os programas nacionais de inovação, já que apenas uma pequena parte das propostas apoiadas é oriunda de instrumentos nacionais, ou mesmo das demais ações do CYTED. Ao mesmo tempo, a inovação de base tecnológica e o comércio de bens e serviços precisam estar coordenados, para haver uma maior efetividade da apropriabilidade dos conhecimentos gerados no âmbito dos agentes ibero-americanos, como forma de geração de riqueza econômica e bem-estar para a população latino-americana.

Ao mesmo tempo, falta uma política de transferência de tecnologia mais efetiva e o uso estratégico de propriedade intelectual, a fim de promover a disseminação do conhecimento e ganhos econômicos aos agentes para canalizar ao desenvolvimento econômico e social.

A crise econômica global pode conferir uma série de restrições aos países ibero-americanos. Por um lado, a redução dos investimentos em C\&T\&I por parte de cada país, o que acarreta na descontinuidade dos investimentos realizados até então. Por outro lado, diminui a demanda efetiva aos produtos e serviços gerados no contexto da economia ibero-americana. Dessa forma, a criação de estratégia que otimize os escassos recursos com resultados mais efetivos é uma condição importante para a geração de riqueza econômica nesta região, o que pode conferir ao Programa CYTED a qualidade de constituir-se em um dos instrumentos para esta articulação. Adicionalmente, deve-se conferir uma maior importância à inovação tecnológica, como potencial gerador de benefícios socioeconômicos na região Ibero-americana, através de iniciativas que sejam capazes de promover a difusão e transferência de tecnologia entre os países que a compõem, e promovendo a criação de novos instrumentos que propiciem um ambiente institucional para a consecução do incremento das atividades cooperativas internacionais, entre as empresas. 


\section{REFERÊNCIAS BIBLIOGRÁFICAS}

COSTA FILHO, Edmilson de Jesus. A dinâmica da cooperação espacial sul-sul: o caso do Programa CBERS (China-Brazil Earth Resources Satellite). Campinas, 2006. Tese (Doutorado). Programa de Pós-graduação em Política Científica e Tecnológica, Instituto de Geociências da Universidade Estadual de Campinas.

LIST, Friedrich. The national system of political economy. English Edition (1904). London: Longman, 1841.

LUNDVALL, Bengt-Ake (ed.). National systems of innovation: towards a theory of innovation and interactive learning. London: Pinter, 1992.

; JOHNSON, Björn; ANDERSEN, Esben Sloth; DALUM, Bent. National systems of production, innovation and competence building. Research Policy, n. 31, p. 213-231, 2002.

NELSON, Richard R. National innovation systems. A comparative analysis. Oxford: Oxford University, 1993.

NICHERA, Antonio Hidalgo; GARRIGÓS, Jose Albors. La internacionalización de la tecnología a través de los proyectos de innovación Iberoeka. Cuadernos de Economía y Dirección de la Empresa, n. 20, p. 57-82, 2004.

PLONSKI, Guilherme Ary. Cooperación empresa - universidad en Iberoamerica: avances recientes. São Paulo: Cyted, 1995.

SABATO, Jorge; BOTANA, Natalio. La ciencia e la tecnología en el desarrollo futuro de América Latina. Revista Integración Latino-americana, p. 15-36, 1968.

SCHUMPETER, Joseph Alois. Teoria do desenvolvimento econômico. São Paulo: Nova Cultural, 1961.

TERUYA, Dirceu Yoshikazu. Globalização tecnológica e o sistema de inovação argentino e brasileiro do setor de equipamentos de telecomunicações. São Paulo, 2004. Tese (Doutorado) — Programa de Pós-graduação em Integração da América Latina da Universidade de São Paulo. 